\title{
Characterization and evaluation of amine-modife
graphene amphotericin B for the treatment of visceral leishmaniasis: in vivo and in vitro studies
}

\author{
This article was published in the following Dove Press journal: \\ Drug Design, Development and Therapy \\ 4 September 2014 \\ Number of times this article has been viewed
}

\author{
Shyam Lal Mudavath' \\ Mahe Talat ${ }^{2}$ \\ Madhukar Rai' \\ Onkar Nath Srivastava ${ }^{2}$ \\ Shyam Sundar' \\ 'Infectious Disease Research \\ Laboratory, Department of Medicine, \\ Institute of Medical Sciences, Banaras \\ Hindu University, Varanasi, India; \\ ${ }^{2}$ Nanoscience Unit, Department of \\ Physics, Banaras Hindu University, \\ Varanasi, India
}

\begin{abstract}
Amphotericin B (AmB) has been the first-line treatment for visceral leishmaniasis (VL), a neglected protozoan disease, especially in regions like Bihar, India, where resistance to antimonials is widespread. However, adverse drug reactions are a major limiting factor. We evaluated a novel formulation of AmB conjugated to amine-modified graphene (f-Gr) for safety and efficacy over conventional AmB. The f-Gr was prepared in a gentle one-step process of noncovalent (amine) functionalization with the help of amino acid L-cysteine. This f-Gr was further conjugated to AmB by peptide bond. The conjugate (f-Gr-AmB) was characterized by Raman spectroscopy, Fourier transform infrared spectroscopy, scanning electron microscopy, and transmission electron microscopy. $\mathrm{f}-\mathrm{Gr}-\mathrm{AmB}$ was found to exhibit lesser cytotoxicity toward J774A. 1 cells than AmB, and did not induce any hepatic or renal toxicity in Swiss albino mice. In vitro antileishmanial assay in J774A.1 cells showed significantly enhanced efficacy of f-GrAmB over AmB. Furthermore, percentage inhibition of amastigote replication in a hamster model of VL was significantly higher in the $\mathrm{f}-\mathrm{Gr}-\mathrm{AmB}$ treated group $(87.8 \%)$ compared to the AmB group (70.4\%). These results suggest that $\mathrm{f}-\mathrm{Gr}-\mathrm{AmB}$ could be a safe and effective alternative to conventional $\mathrm{AmB}$ in the treatment of VL.
\end{abstract}

Keywords: antileishmanial, efficacy, cytotoxicity, macrophage, Raman spectroscopy, amastigote

\section{Introduction}

Visceral leishmaniasis (VL) is one of the most neglected tropical diseases caused by intracellular protozoan parasites of the Leishmania donovani complex. VL is the second most common cause of mortality and fourth most common cause of morbidity due to tropical diseases, causing 20,000 to 40,000 deaths annually. ${ }^{1}$ The treatment options for VL are highly circumscribed, and if untreated, the disease most often culminates in death. ${ }^{2,3}$ Amphotericin B (AmB) is used as a first-line drug for VL in India and constitutes an alternate treatment for patients resistant to antimonials elsewhere. However, it has major drawbacks in terms of protracted hospitalization and significant nephrotoxicity. ${ }^{4,5}$ In spite of the fact that lipid formulations of $\mathrm{AmB}$ exhibit better therapeutic indexes, their use is throttled by higher costs and their intravenous route of administration. ${ }^{6,7}$

The variable efficacy of certain antileishmanial drugs and significant toxicity of others has been a motivation to explore novel therapeutic strategies. ${ }^{8}$ One area of concern is the localization of the parasite within the lysosomes of reticuloendothelial cells, which hampers the access and thus bioavailability of antileishmanial drugs. ${ }^{9}$ Hence, the development of delivery systems that can target macrophages intracellularly is crucial, and
Correspondence: Shyam Sundar, Infectious Disease Research Laboratory, Department of Medicine, Institute of Medical Sciences, Banaras Hindu University, Varanasi, Uttar Pradesh, 22I 005, India

$\mathrm{Tel}+915422369632$

Fax + 9I 5422367568

Email drshyamsundar@hotmail.com 
could potentially lead to new treatment paradigms for VL with improved therapeutic efficacy and reduced toxicity. ${ }^{10}$ Nanomaterials have proved to be valuable as vectors for intracellular drug delivery. They fall into a size range close to that of proteins and other biological macromolecules, which makes it possible for them to interact with and enter cells. ${ }^{11}$ Previously, we described that nanoparticles of AmB have significantly greater efficacy than conventional AmB, with an improved safety profile. ${ }^{12}$ Subsequently, we also reported functionalized carbon nanotubes (f-CNTs) as an effective nanovector for AmB for oral administration in the treatment of VL. ${ }^{13,14}$

Graphene and its derivatives are now at the forefront of nearly every rapidly developing field of science and engineering, including biomedical applications such as biosensor development, imaging, drug delivery, bacterial inhibition, and photothermal therapy. ${ }^{15-19}$ The fascinating physicochemical properties and low cost, scalable production coupled with an intrinsic biocompatibility, and facile biofunctionalization make graphene an attractive nanoscaffold for drug delivery. ${ }^{20,21}$ Functionalized graphene oxide has been shown to be a promising nanovector for the efficient delivery of drugs into cells. Despite the great application potential, it is important to mention that graphene itself possesses zero band gap as well as inertness to reaction. The $\pi$ - $\pi$ stacking between graphene sheets (GS) results in the formation of multilayers. Pristine GS are hydrophobic in nature, and tend to aggregate in solution, which is driven by enhanced van der Waals forces; this feature gives rise to technical difficulties during application. ${ }^{22}$ This is one of the reasons that an increasing number of reports are available on functionalization of graphene and its derivatives. Thus, functionalization of GS is important for their future applications. Similar to the drug loading on CNTs, the graphene surface with delocalized p-electrons can be utilized for effective loading of drugs via $\pi-\pi$ stacking. GS as a drug carrier are interesting because both sides of a single sheet could be accessible for drug binding. The extremely large surface area of graphene $\left(\sim 2,630 \mathrm{~m}^{2} / \mathrm{g}\right)$, with every atom exposed on its surface, allows for ultra-high drug loading efficiency on graphene as compared to CNT, the surface area of which is almost half that of graphene. ${ }^{23}$

However, the growing concern about cytotoxicity of graphene oxide has drawn researchers to look for alternate derivatives. ${ }^{24-26}$ Amine-functionalized graphene was shown to be a safer alternative to graphene oxide, with potential for biomedical applications. ${ }^{27}$ Hence, in the present study we explore the efficacy and toxicity of a novel AmB formulation as a conjugate (f-Gr-AmB) with amine-modified graphene (f-Gr) in the treatment of VL.

\section{Materials and methods}

\section{Parasites for infection and cell lines}

The J774A.1 macrophage cell line (obtained from the National Centre for Cell Science, Pune, India) was used as a cellular host. World Health Organization (WHO) reference strain of L. donovani LEM 138 (MHOM/IN/00/DEVI) was used for raising infection. Parasites were cultured in a supplemented Roswell Park Memorial Institute (RPMI) 1640 medium (Thermo Fisher Scientific, Waltham, MA, USA), as described previously. ${ }^{28}$

\section{Animals}

Male Syrian golden hamsters, Mesocricetus auratus (45-50 g), were procured from the animal house facility of the Central Drug Research Institute (CDRI), Lucknow, India for studying in vivo antileishmanial activity, and male Swiss albino mice (30-40 g) for in vivo toxicity studies were purchased from Central Animal Facility, Institute of Medical Sciences (IMS), Banaras Hindu University (BHU), Varanasi, India. All experiments were performed using protocols approved by the Central Animal Ethics Committee (CAEC), IMS, BHU (CAEC number Dean/2014/CAEC/615). The guidelines of the Council for the Purpose of Control and Supervision of Experiments on Animals (CPCSEA), Ministry of Environment and Forests, Government of India were followed.

\section{Synthesis of GS}

The GS were synthesized by thermal exfoliation of graphite oxide (GO). GO was prepared using graphite powder according to a slightly modified Staudenmaier's method. ${ }^{29}$ In a typical experiment, graphite powder $(<50 \mu \mathrm{m}, 1 \mathrm{~g})$ was treated with a strong oxidizing solution of $18 \mathrm{~mL}$ concentrated analytical research grade $\mathrm{H}_{2} \mathrm{SO}_{4}$ (Sigma-Aldrich Co, St Louis, MO, USA), $9 \mathrm{~mL}$ concentrated analytical research grade $\mathrm{HNO}_{3}$ (Sigma-Aldrich $\mathrm{Co}$ ), and $11 \mathrm{~g}$ potassium chlorate (less than 99\% pure; Merck and Co, Inc., Whitehouse Station, NJ, USA) at room temperature for 5 days under magnetic stirring conditions. As-obtained GO solution was washed with distilled water and $10 \% \mathrm{HCl}$ solution to remove sulfate and other ion impurities. Chemical purification was done to remove impurities that exist or are intercalated between the various graphite planes. The $\mathrm{GO}$ powder was dried at $80^{\circ} \mathrm{C}$ under vacuum. As-prepared GO ( $200 \mathrm{mg})$ was thermally exfoliated by rapid heating at $1,050^{\circ} \mathrm{C}$ for 30 seconds under an argon atmosphere. The product was isolated by filtration, washed copiously with water, and vacuum dried. 


\section{Amine functionalization of GS}

GS were functionalized with the amino acid L-cysteine in double distilled water. A mass of $25 \mathrm{mg}$ of GS was added to $250 \mathrm{~mL}$ double distilled water and sonicated for 1.5 hours at room temperature to obtain a homogeneous solution. Then, $0.1 \mathrm{M} \mathrm{L}$-cysteine (in double distilled water) was added to the solution and sonicated for 30 minutes, followed by 3 hours of constant magnetic stirring. The f-Gr so obtained was thoroughly washed with double distilled water in a centrifuge at $10,000 \mathrm{rpm}$ for 10 minutes, and the solution phase was discarded. This washing was repeated five times to remove any unbound L-cysteine. $\mathrm{f}-\mathrm{Gr}$ was dried in ambient atmosphere and stored at $4^{\circ} \mathrm{C}$. Since this method is gentle and does not require any harsh chemicals such as acids, it is preferred for targeted drug delivery, biosensing, and other applications using enzymes. ${ }^{30-32}$

\section{Attachment of AmB to $\mathrm{f}-\mathrm{Gr}$}

$\mathrm{f}-\mathrm{Gr}$ was dispersed in deionized water, and $\mathrm{AmB}$ powder was dissolved in dimethyl sulfoxide. The AmB solution was mixed with f-Gr solution separately and sonicated in an ultrasonication bath at room temperature for about 24 hours, followed by $\sim 4$ hours of magnetic stirring. The solution was centrifuged and washed with double distilled water approximately three to four times to remove unbound $\mathrm{AmB}$. By comparing the optical density (OD) of known concentrations of $\mathrm{AmB}$ to the OD of unattached, dissolved $\mathrm{AmB}$ remaining in the supernatant of $\mathrm{f}-\mathrm{Gr}-\mathrm{AmB}, \mathrm{AmB}$ loaded to $\mathrm{f}-\mathrm{Gr}$ was calculated as

$$
\text { Loading efficiency }=(\mathrm{OD} 1-\mathrm{OD} 2) /(\mathrm{OD} 1)^{*} 100 \text {, }
$$

where OD1 $=\mathrm{A}_{409}$ of $\mathrm{AmB}(1 \mathrm{mg} / \mathrm{mL})$, and OD2 $=\mathrm{A}_{409}$ of unattached $\mathrm{AmB}$ from the supernatant.

\section{Characterization techniques}

The ultrastructures of graphene and its derivatives (GS, $\mathrm{f}-\mathrm{Gr}$, and $\mathrm{f}-\mathrm{Gr}-\mathrm{AmB}$ ) were examined by scanning electron microscope (SEM; a Philips XL 20; Koninklijke Philips NV, Amsterdam, the Netherlands or a Quanta 200; FEI, Hillsboro, OR, USA) and transmission electron microscope (TEM; Technai $20 \mathrm{G}^{2}$, FEI). Functionalization of AmB was established by Fourier transform infrared (FTIR) spectroscopy using a Spectrum 100 spectrometer (PerkinElmer, Waltham, MA, USA) with the help of universal attenuated total reflectance attachment. Attachment of AmB to f-Gr was established by Raman spectroscopy, using an HR-800 Raman spectrometer for argon ion laser $(\lambda=514 \mathrm{~nm}$; Horiba Jobin Yvon, Kyoto, Japan).

\section{In vitro cytotoxicity testing}

The cytotoxicity of f-Gr-AmB, f-Gr, and AmB was assessed as described previously. ${ }^{12}$ J774A.1 macrophage cells were plated in a $96-$ well microplate at a density of $2.5 \times 10^{4}$ cells/ well. Then, the cells were incubated in triplicate with varying concentrations of f-Gr-AmB $(0.005-0.64 \mu \mathrm{g} / \mathrm{mL})$, f-Gr $(0.0625-8.0 \mu \mathrm{g} / \mathrm{mL})$, or AmB $(0.005-0.64 \mu \mathrm{g} / \mathrm{mL})$ for 72 hours at $37^{\circ} \mathrm{C}, 5 \% \mathrm{CO}_{2}$; untreated cells served as a control. Cytotoxicity was assessed using the colorimetric MTT (3-[4,5-Dimethylthiazol-2-yl]-2,5-diphenyltetrazoliumbromide) reduction assay. The $50 \%$ cytotoxicity concentration $\left(\mathrm{CC}_{50}\right)$ was calculated from the graph of ODs plotted against drug concentration, taking OD of the control well as a measure of $100 \%$ survival.

\section{In vitro antileishmanial activity against intracellular amastigotes}

The J774A.1 macrophage cells were seeded at a density of $5 \times 10^{4}$ cells/well on 8-chamber Lab Tek tissue culture slides (USA Scientific, Inc., Ocala, FL, USA) and were allowed to adhere for 2 hours in a $\mathrm{CO}_{2}$ incubator with $5 \% \mathrm{CO}_{2}$ at $37^{\circ} \mathrm{C}$. The wells were then washed twice with serum-free RPMI 1640 medium to remove non-adherent macrophages. Further, adhered macrophages were infected with metacyclic stage parasites of $L$. donovani LEM 138, maintaining a Leishmania: macrophage ratio of $10: 1$ in a $200 \mu \mathrm{L}$ final solution of a complete RPMI 1640 medium supplemented with $15 \%(\mathrm{v} / \mathrm{v})$ heat inactivated fetal bovine serum (Thermo Fisher Scientific) and $50 \mathrm{mg} / \mathrm{L}$ gentamicin overnight. After 24 hours, free promastigotes were washed with serum-free medium, and infected macrophages were incubated with AmB $(0.005-0.02 \mu \mathrm{g} / \mathrm{mL}), \mathrm{f}-\mathrm{Gr}(0.005-0.02 \mu \mathrm{g} / \mathrm{mL})$, and f-Gr-AmB $(0.00125-0.005 \mu \mathrm{g} / \mathrm{mL})$ in duplicate for 72 hours with $5 \% \mathrm{CO}_{2}$ at $37^{\circ} \mathrm{C}$, except in the control well (without drug), followed by methanol fixing for 1 minute and staining with giemsa (Qualigens, Mumbai, India). At least 100 macrophage nuclei were counted per well for calculating the percentage of infected macrophages and the number of amastigotes per 100 macrophages. The $\mathrm{IC}_{50}$ (concentration of drug that inhibits $50 \%$ of $L$. donovani amastigotes) of each drug was calculated, as described previously. ${ }^{12}$

\section{In vivo antileishmanial studies}

Hamsters are an optimal model of $L$. donovani infection, as they mimic several clinical and immunopathological aspects of human VL and can withstand repeated spleen biopsies to check parasite burden. Twenty-five male hamsters (4-6 weeks of age) were infected by intracardiac injection of $1 \times 10^{7}$ 
promastigotes of L. donovani LEM 138. After 30 days, infection was confirmed by giemsa staining of splenic smears from four randomly selected animals. AmB, f-Gr, and f-Gr-AmB were reconstituted for in vivo administration in $1 \times$ phosphate buffered saline (PBS) at a concentration of $1.5 \mathrm{mg} / \mathrm{mL}$. The treatment and control groups consisted of 4-6 animals in each group, and treatment animals were treated intraperitoneally with $\operatorname{AmB}(n=10), f-G r(n=10)$, and f-Gr-AmB $(n=12)$ at a dose of $5 \mathrm{mg} / \mathrm{kg}$ body weight for 5 consecutive days, while the control group $(n=8)$ received an equal volume of PBS. Autopsies were conducted on day 7 post-treatment. The weight of the spleen was measured immediately after autopsy, and dabbed imprints on glass slides were prepared. The parasite burden, percentage of inhibition of splenic parasite load, and the percentage of suppression of parasite replication were calculated. ${ }^{13}$ This experiment was repeated with 25 male hamsters for reproducibility.

\section{In vivo toxicity studies}

The in vivo toxicity of f-Gr-AmB, f-Gr, and AmB was assessed in 40 Swiss albino mice ( $25 \pm 5$ weeks of age) with a 5-day course of daily intraperitoneal injection using $5 \mathrm{mg} / \mathrm{kg}$, $10 \mathrm{mg} / \mathrm{kg}$, and $20 \mathrm{mg} / \mathrm{kg}$ dose regimens in 12 mice with 4 mice for each concentration. Aspartate transaminase (AST), alanine aminotransferase (ALT), urea, and creatinine levels were used as biochemical markers to evaluate hepatotoxicity and nephrotoxicity. The control group, comprising 4 mice, was injected with PBS. After a 5-day course, the mice were euthanized, blood was drawn, and serum was separated. Levels of ALT, AST, urea, and creatinine were estimated to assess renal and hepatic function using commercially available kits (Autozyme GPT; Accurex Biomedical Pvt. Ltd., Mumbai, India. Enzopak SGOT; Reckon Diagnostics Pvt. Ltd., Gujart, India. Lyphozyme Urea Berthelot; Beacon Diagnostics Pvt. Ltd., Gujarat, India. Autozyme creatinine; Accurex Biomedical Pvt. Ltd., Mumbai, India).

\section{Statistical analysis}

GraphPad Prism version 5 was used to calculate the $\mathrm{CC}_{50}$ and $\mathrm{IC}_{50}$ values. An unpaired $t$-test (two-tailed) was applied to determine the significance of the differences between the cytotoxicity and antileishmanial activity of the two drugs, AmB and f-Gr-AmB.

\section{Results}

Schematic representations of functionalization of GS and attachment of AmB are shown in Figure 1. Representative TEM images of GS, f-Gr, and f-Gr-AmB samples are shown

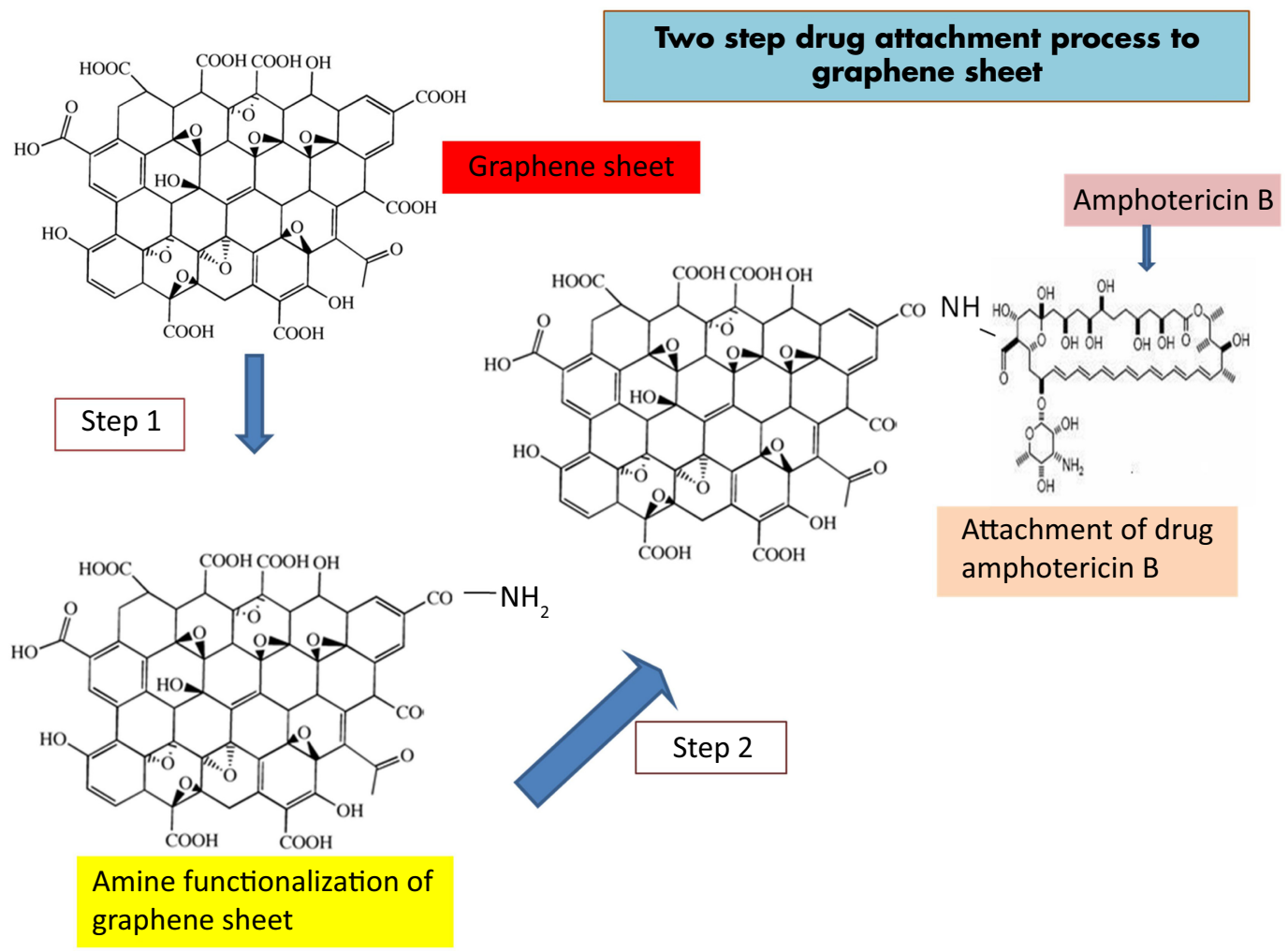

Figure I Schematic representation of amphotericin B attachment to functionalized graphene sheet. 

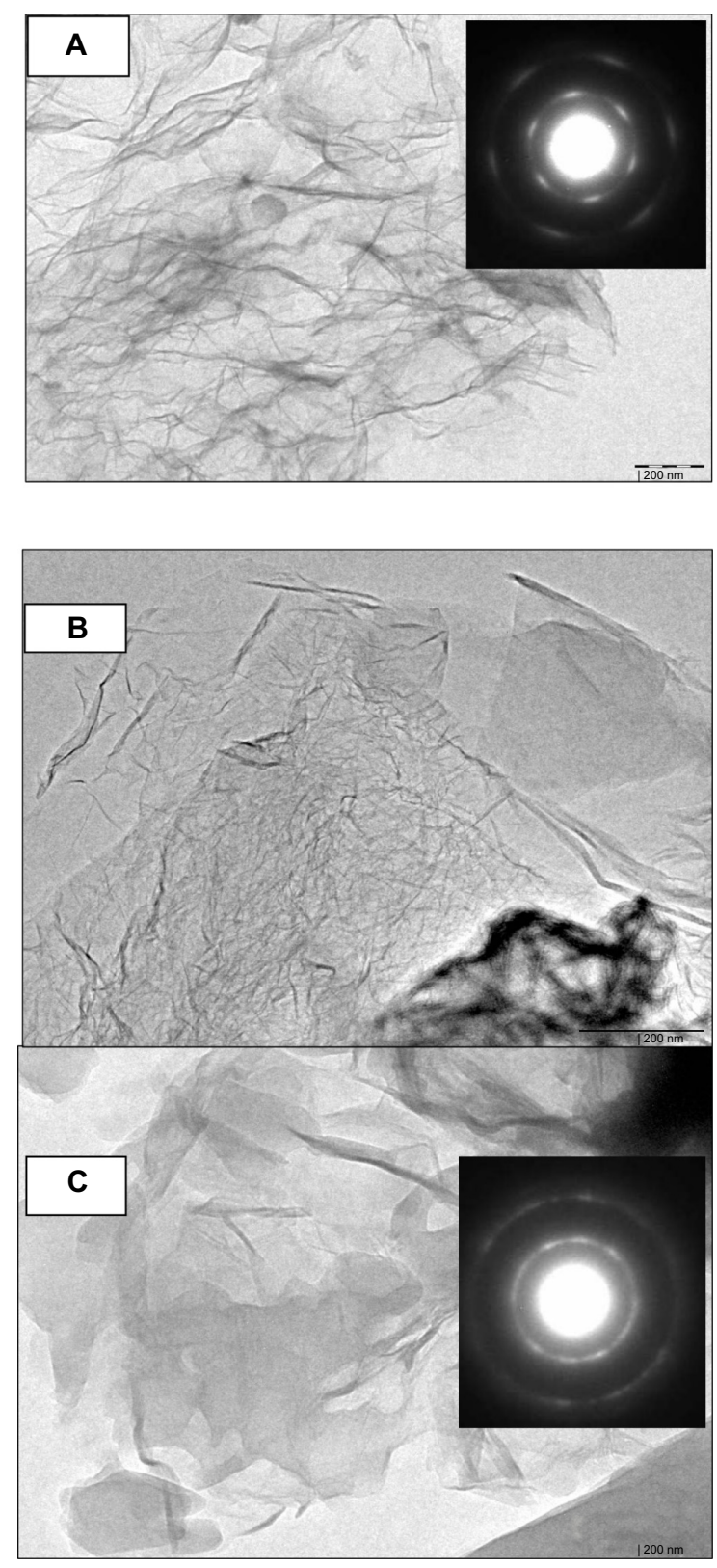

Figure 2 TEM images of GS (A), f-Gr (B), and f-Gr-AmB (C).

Notes: The TEM image of GS shows a wrinkled paper-like structure $(\mathbf{A})$, which appears to be very thin. The inset is the selected area electron diffraction pattern (SAED) of GS, showing clear diffraction spots; the diffraction spots were indexed to a hexagonal graphite crystal structure. In contrast, the TEM image of $\mathrm{f}-\mathrm{Gr}$ (B) shows a smooth surface, which is due to functionalization by L-cysteine. AmB deposited on $\mathrm{f}-\mathrm{Gr}(\mathbf{C})$ appears as a cloudy shadow. The SAED pattern of the inset demonstrates the well-textured, crystalline nature of $\mathrm{f}-\mathrm{Gr}-\mathrm{AmB}$, which supports the conclusion that $A m B$ is attached.

Abbreviations: TEM, transmission electron microscopy; GS, graphene sheets; $f-G r$, amine-modified graphene; $A m B$, amphotericin B; $f-G r-A m B$, a novel $A m B$ formulation as a conjugate with $\mathrm{f}-\mathrm{Gr}$.

in Figure 2. The TEM image of GS reveals a wrinkled paperlike structure, which appears to be very thin (Figure 2A). The Figure 2A inset is the selected area electron diffraction pattern of GS, showing clear diffraction spots. The diffraction spots were indexed to hexagonal graphite crystal structure. In contrast, the TEM image of f-Gr shows a smoothened surface, which is due to the functionalization by L-cysteine (Figure 2B). The image of Figure 2C shows AmB deposited on $\mathrm{f}-\mathrm{Gr}$, and appears as a cloudy shadow. The selected area electron diffraction pattern of the inset in Figure $2 \mathrm{C}$ demonstrates the well-textured and crystalline nature of $\mathrm{f}-\mathrm{Gr}-\mathrm{AmB}$, which supports the conclusion that $\mathrm{AmB}$ is attached.

The surface morphologies of GS, f-Gr, and f-Gr-AmB were examined by SEM in secondary electron imaging mode. The random orientation and wavy appearance of exfoliated GO is seen from the SEM images. The top views of f-Gr clearly illustrate the typically flake-like structure with slightly scroll-edged shapes (Figure S1A). It is clearly visible from Figure S1B that the small L-cysteine molecules are closely anchored on the surface of GS and held noncovalently (marked with the arrow). Figure S1C presents the SEM image of f-Gr-AmB. It obviously shows that a layer of AmB was formed homogeneously like a blanket (rectangular area) on the $\mathrm{f}-\mathrm{Gr}$ surface, confirming that the AmB molecule is uniformly attached on the GS.

\section{FTIR analysis of GS, $f-G r$, and $f-G r-A m B$}

A representative FTIR spectrum of GO, f-Gr, and f-Gr-AmB is shown in Figure S2A-C. It may be mentioned here that GS prepared from thermal exfoliation of $\mathrm{GO}$ at $1,050^{\circ} \mathrm{C}$ for 30 seconds invariably contains some oxygen functionalities. These are $\mathrm{C}=\mathrm{O}, \mathrm{COOH}$, and $\mathrm{OH}$ functionalities, which are revealed by FTIR. $\mathrm{C}=\mathrm{C}$ stretching at $1,558 \mathrm{~cm}^{-1}$ is attributed to the vibration of the carbon skeleton of $\mathrm{GS} ; \mathrm{C}=\mathrm{O}$ stretching occurring at $1,748 \mathrm{~cm}^{-1}$ is also recorded. The observed peak in Figure S2A at 3,411 $\mathrm{cm}^{-1}$ corresponds to hydroxyl $(-\mathrm{OH})$ stretching and vibration. In addition, the peaks at 1,398 and $1,120 \mathrm{~cm}^{-1}$ are related to $\mathrm{C}-\mathrm{OH}$ (hydroxyl) and $\mathrm{C}-\mathrm{O}$ (epoxy), respectively. This observation confirms the presence of oxygen containing functional groups, eg, $\mathrm{C}=\mathrm{O}, \mathrm{C}-\mathrm{OH}$, and $\mathrm{C}-\mathrm{O}$ on the GS. It is generally recognized that the hydroxyl and epoxy groups are present above and below the basal planes, while the carboxylic groups are bound to the edge of GS. ${ }^{33}$ In $\mathrm{f}-\mathrm{Gr}$ (Figure S2B), an additional peak is observed at $3,016 \mathrm{~cm}^{-1}$, which is due to the stretching vibrations of an alkane group, while the presence of a peak at $1,475 \mathrm{~cm}^{-1}$ can be assigned to $\mathrm{N}-\mathrm{H}$ stretching vibrations of the amine group, and the $1,385 \mathrm{~cm}^{-1}$ peak is due to the $\mathrm{C}-\mathrm{N}$ stretching vibrations. In Figure S2C, the peak at $1,902 \mathrm{~cm}^{-1}$ is due to the $\mathrm{C}-\mathrm{O}$ stretching of $\mathrm{f}-\mathrm{Gr}$. The peak at $1,019 \mathrm{~cm}^{-1}$ is associated with the stretching vibration mode of the hydroxyl group of the carboxylic group present in $\mathrm{AmB}$, which reacts with the 
amine group of the $\mathrm{f}-\mathrm{Gr}$. A new peak at $1,609 \mathrm{~cm}^{-1}$ represents amide carbonyl and results from the $\mathrm{C}=\mathrm{O}$ stretching along with the $\mathrm{N}-\mathrm{H}$ deformation mode. ${ }^{34}$

\section{Raman spectra of GS and f-Gr-AmB}

Figure $3 \mathrm{~A}$ shows the Raman spectra of GS. The characteristic peak at $1,308 \mathrm{~cm}^{-1}$ of $\mathrm{D}$ (disorder) peak, $1,589 \mathrm{~cm}^{-1}$ of $\mathrm{G}$ (graphite) peak, and 2D peak at 2,661 $\mathrm{cm}^{-1}$ confirm the formation of GS. ${ }^{35}$ The intensity of $\mathrm{D} / \mathrm{G}$ ratio is found to be $\sim 0.82$. Figure $3 \mathrm{~B}$ shows the Raman spectra of $\mathrm{AmB}$ attached to $\mathrm{f}-\mathrm{Gr}$. Along with the almost eliminated $\mathrm{D}$ peak at $1,381 \mathrm{~cm}^{-1}$ and the $\mathrm{G}$ peak at $1,565 \mathrm{~cm}^{-1}$, two more peaks are appearing in the spectra, ie, at $1,012 \mathrm{~cm}^{-1}$ and at $1,156.78$ $\mathrm{cm}^{-1}$. These are most probably due to the AmB attachment with f-Gr. A huge red shift of $25 \mathrm{~cm}^{-1}$ in the $G$ peak is due to the attachment of AmB to the surface of f-Gr. The decreased value of the $\mathrm{I}_{\mathrm{D}} / \mathrm{I}_{\mathrm{G}}$ ratio, ie, a decrease of $\sim 0.404$, is due to the reduced intensity of $D$ peak and the sharp $G$ peak, which signify that the AmB attached on the f-Gr is decreasing the defects of the system. It can be assumed that
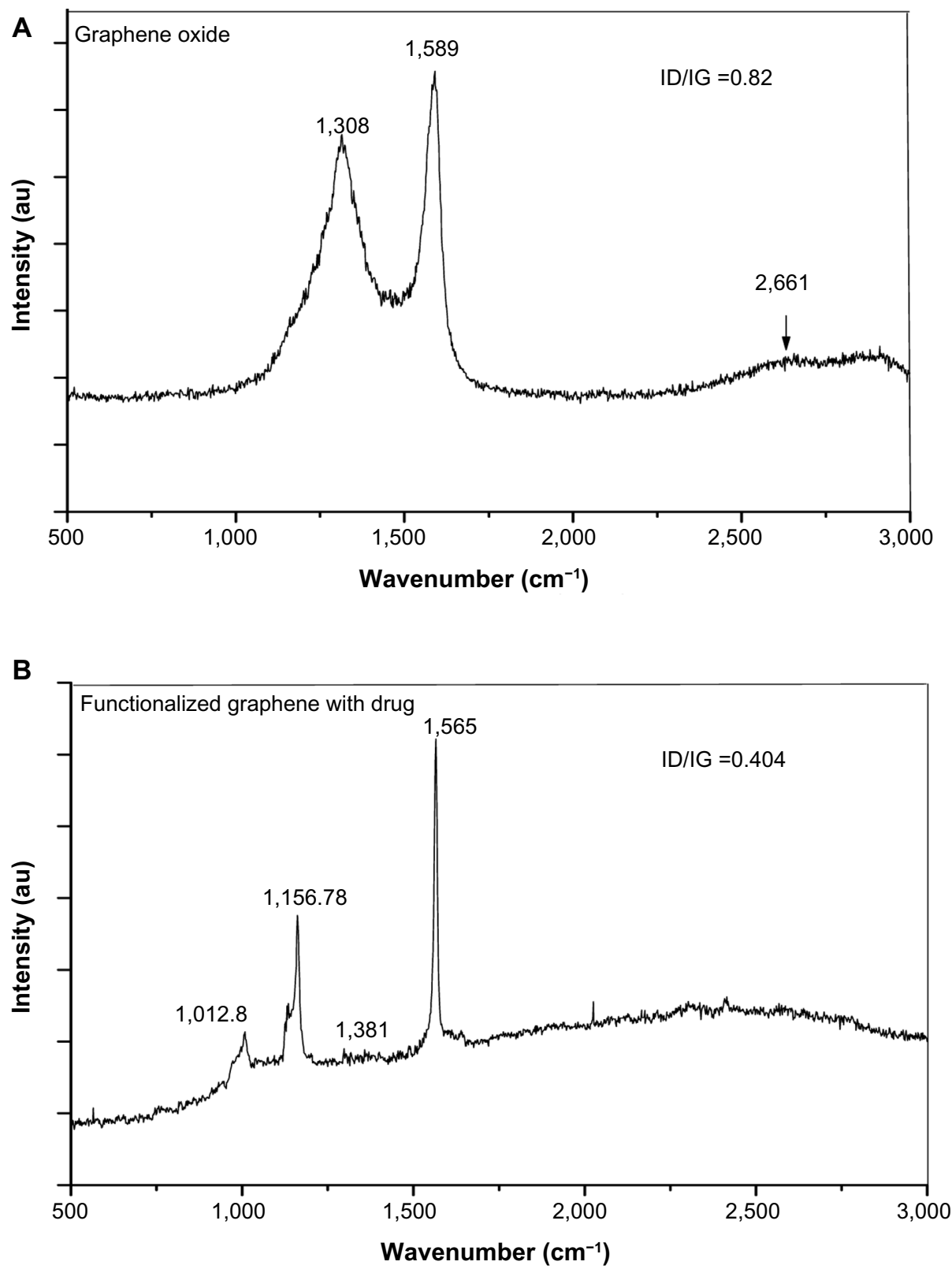

Figure 3 Raman spectra of GS (A) and $\mathrm{f}-\mathrm{Gr}-\mathrm{AmB}(\mathbf{B})$.

Notes: (A) The characteristic peak at $1,308 \mathrm{~cm}^{-1}$ of D peak, I,589 $\mathrm{cm}^{-1}$ of $G$ peak and 2D peak at 2,66I $\mathrm{cm}^{-1}$ confirms the formation of graphene. (B) Two more peaks are appearing in the spectra ie, at $1,012 \mathrm{~cm}^{-1}$ and at $1,156 \mathrm{~cm}^{-1}$ due to the attachment of amphotericin $B$ along with the almost eliminated $D$ peak at $I, 308 \mathrm{~cm}^{-1}$.

Abbreviations: GS, graphene sheets; f-Gr-AmB, a novel amphotericin B formulation as a conjugate with amine-modified graphene; I, intensity of; D, disorder peak; G, graphite peak. 
due to a masking effect of the AmB, the defects of the $\mathrm{f}-\mathrm{Gr}$ are reduced, which is apparently visible in the spectra. So, it can be concluded that AmB is effectively attached on the surface, or most probably on the edges, of the f-Gr.

The OD of a known concentration of AmB $(1 \mathrm{mg} / \mathrm{mL})$ and unattached AmB from the supernatant of f-Gr-AmB, were 3.392 and 0.868 at $409 \mathrm{~nm}$, respectively. The loading efficiency of $74.39 \%$ was consistent with no observable $\mathrm{f}$-Gr-AmB precipitation during 24 hours of storage at room temperature.

\section{f-Gr-AmB showed better antileishmanial activity without an increased cytotoxicity toward macrophages}

Taking into account the improved antileishmanial activity of $\mathrm{AmB}$, as a result of conjugation to $\mathrm{f}-\mathrm{Gr}$, we looked into whether this improvement is associated with increased toxicity for macrophages. The $\mathrm{CC}_{50}$ of the compounds against the J774A.1 cell line were: AmB, 0.375 $\pm 0.06 \mu \mathrm{g} / \mathrm{mL}$; f-Gr-AmB, $0.52 \pm 0.02 \mu \mathrm{g} / \mathrm{mL}$; and f-Gr, $4.28 \pm 1.27 \mu \mathrm{g} / \mathrm{mL}$ $(P=0.04)$. This $\mathrm{CC}_{50}$ of $\mathrm{f}-\mathrm{Gr}-\mathrm{AmB}$ is far higher than the $\mathrm{IC}_{50}$ $(0.003 \mu \mathrm{g} / \mathrm{mL})$ against intramacrophage amastigotes. It is important to note that f-Gr-AmB does not show any significant toxic effects in vitro on J774A.1 macrophage cells at antileishmanial concentrations. These data represent the mean \pm standard deviation of two experiments performed in triplicate.

\section{f-Gr-AmB showed greater inhibition of intramacrophage amastigotes}

Amastigotes, the infective form of Leishmania, reside and replicate in the reticuloendothelial macrophages. Reduction in the number of intracellular amastigotes of infected macrophages is indicative of better antileishmanial activity of a drug formulation. The antileishmanial activity of the compounds was: AmB, $\mathrm{IC}_{50}=0.027 \pm 0.004 \mu \mathrm{g} / \mathrm{mL}$; and f-Gr$\mathrm{AmB}, \mathrm{IC}_{50}=0.003 \pm 0.0004 \mu \mathrm{g} / \mathrm{mL}$. The $\mathrm{IC}_{50}$ of $\mathrm{f}-\mathrm{Gr}-\mathrm{AmB}$ was nine times more than $\mathrm{AmB}$ against intramacrophage amastigotes $(P=0.003)$. This gives substance to the conclusion that $\mathrm{f}-\mathrm{Gr}-\mathrm{AmB}$ is a better antileishmanial formulation compared to conventional AmB.

\section{f-Gr-AmB showed improved antileishmanial activity in the hamster model of VL}

For analysis, the outcome from both the sets of in vivo experiments was conflated to yield a mean value. No significant difference was observed in the weight of both control and treatment group hamsters. There was a substantial improvement in the antileishmanial activity of f-Gr-AmB compared to conventional AmB. The percentage inhibition of parasites in the spleens of f-Gr-AmB treated Leishmania-infected hamsters $(87.8 \%)$ was higher when compared with AmB treated Leishmania-infected hamsters $(70.4 \%)(P=0.002$; Table 1$)$. In contrast, $\mathrm{f}-\mathrm{Gr}$ showed a negligible antileishmanial activity $(4.96 \%)$. There was a more significant reduction in the parasite burden in the

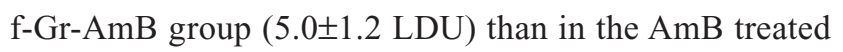
group (16.9 $\pm 2.5 \mathrm{LDU})(P=0.005$; Table 1$)$. The percentage suppression of parasite replication of $\mathrm{f}-\mathrm{Gr}-\mathrm{AmB}$ treated Leishmania-infected hamsters $(90.3 \%)$ was higher when compared with AmB treated Leishmania-infected hamsters $(75.9 \%)(P=0.001$; Table 1$)$. Furthermore, there was a greater reduction in the spleen size of $\mathrm{f}-\mathrm{Gr}-\mathrm{AmB}$ treated hamsters $(2.6 \mathrm{~cm})$ than in infected controls $(3.4 \mathrm{~cm})$, suggestive of a decrease in parasite burden (Figure 4).

Table I In vivo efficacy studies in Syrian golden hamsters

\begin{tabular}{|c|c|c|c|c|c|c|}
\hline Group & $\begin{array}{l}\text { Before } \\
\text { treatment } \\
n=8\end{array}$ & $\begin{array}{l}\text { f-Gr-AmB } \\
\text { (group I) } \\
n=12\end{array}$ & $\begin{array}{l}\text { AmB } \\
\text { (group 2) } \\
n=10\end{array}$ & $\begin{array}{l}\mathrm{f}-\mathrm{Gr} \\
\text { (group 3) } \\
\mathrm{n}=10\end{array}$ & $\begin{array}{l}\text { Control } \\
\text { (group 4) } \\
n=8\end{array}$ & $P$-value \\
\hline Spleen weight (gm) & $1.02 \pm 0.1$ & $0.98 \pm 0.07$ & $1.1 \pm 0.1$ & $1.0 \pm 0.1$ & $1.3 \pm 0.17$ & 0.01 \\
\hline Mean \pm SD & & & & & & \\
\hline Amastigotes/500 nuclei & $504 \pm 40$ & $66 \pm 11.5$ & $152 \pm 29$ & $478.5 \pm 12.1$ & $634 \pm 29$ & 0.002 \\
\hline $\begin{array}{l}\text { Parasite burden }\left(\mathrm{LDU}^{\mathrm{b}}\right) \\
\text { post-treatment } \times 10^{4}\end{array}$ & $51.9 \pm 8.2$ & $5.0 \pm 1.2$ & $16.9 \pm 2.5$ & $52.4 \pm 7.6$ & $85.5 \pm 9.3$ & 0.005 \\
\hline $\begin{array}{l}\text { Percentage inhibition } \\
\text { of splenic parasite load }\end{array}$ & & $87.8 \pm 4.6$ & $70.4 \pm 4.4$ & & & 0.002 \\
\hline $\begin{array}{l}\text { Percentage suppression } \\
\text { of parasite replication }\end{array}$ & & $90.3 \pm 3.7$ & $75.9 \pm 3.7$ & & & 0.001 \\
\hline
\end{tabular}

Notes: aP-value between group I (f-Gr-AmB) and group 2 (AmB); ${ }^{b}$ number of amastigotes per 500 nuclei $\times$ tissue weight $(\mathrm{mg})$.

Abbreviations: $n$, number of hamsters; $A m B$, amphotericin B; $f-G r$, amine-modified graphene; $f-G r-A m B$, a novel AmB formulation as a conjugate with $f-G r ; ~ S D$, standard deviation. 


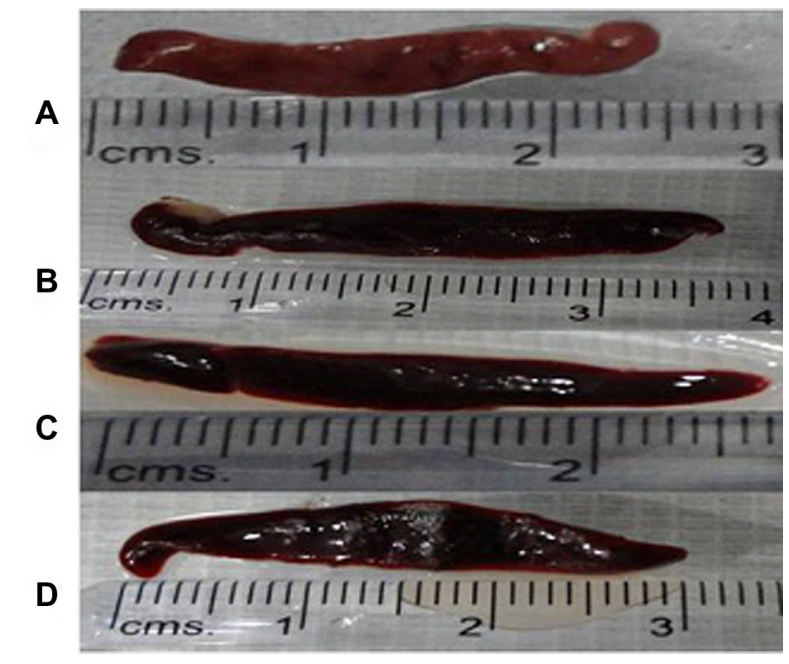

Figure 4 Spleen sizes of hamsters of different experimental groups.

Notes: (A) Healthy control $(2.4 \mathrm{~cm})$; (B) vehicle control $(3.4 \mathrm{~cm}) ;($ C) $\mathrm{f}-\mathrm{Gr}-\mathrm{AmB}$ treated $(2.6 \mathrm{~cm})$; and $(\mathbf{D}) \mathrm{AmB}$ treated $(3 \mathrm{~cm})$.

Abbreviations: AmB, amphotericin B; $f-G r-A m B$, a novel $A m B$ formulation as a conjugate with amine-modified graphene.

\section{f-Gr-AmB did not induce hepatorenal toxicity in Swiss albino mice}

Adverse drug reactions are major limiting factor of AmB. ${ }^{36}$ Hence, we looked for the potential in vivo toxicity of the formulations in Swiss albino mice. No obvious signs of toxic effects for AmB, f-Gr, and $\mathrm{f}-\mathrm{Gr}-\mathrm{AmB}$ injected mice at a dose of up to $20 \mathrm{mg} / \mathrm{kg}$ within 5 days were noted. Neither significant body weight drop nor death was noted in any treatment group. No noticeable difference was observed from blood serum biochemistry of renal and hepatic enzymes, urea (range, 13.4-18.5 mg/dL), creatinine (range, $0.4-0.56 \mathrm{mg} / \mathrm{dL}$ ), AST (range, 31.7-52.2 IU/L), or ALT (range, 33.8-46.3 IU/L) between the control and treatment groups at all doses, and all were within the normal reference range (Table 2).

\section{Discussion}

The present study was designed to evaluate graphene-based drug delivery of AmB for its toxicity, antileishmanial efficacy, and cost effectiveness. Drug delivery is one of the most extensively explored applications of carbon nanomaterials in biomedicine. ${ }^{37}$ During attachment, the carboxylic group of $\mathrm{AmB}$ reacts with the amine group of $\mathrm{f}-\mathrm{Gr}$, forming a stable f-Gr-AmB conjugate. Being a covalent bond, the drug is strongly bonded to the f-Gr and does not dissociate during delivery of the drug AmB.

The f-Gr-AmB conjugate exhibited significantly enhanced activity against intracellular amastigotes of $L$. donovani in a macrophage model with 9-fold improvement in $\mathrm{IC}_{50}$ values

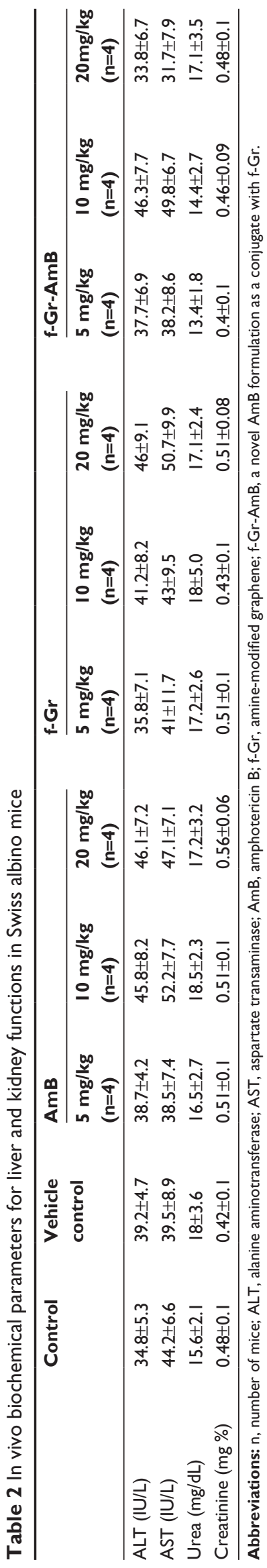


over conventional AmB. This may be attributed to more efficient intracellular uptake of $\mathrm{f}-\mathrm{Gr}-\mathrm{AmB}$ by macrophages, and hence better delivery of AmB to amastigotes.

These results were corroborated in a golden hamster model of $L$. donovani infection, where significantly greater inhibition was observed with f-Gr-AmB. Treatment of infected hamsters with $\mathrm{f}-\mathrm{Gr}-\mathrm{AmB}$ induced a remarkable decrease in parasitic load and amelioration of VL pathology compared to AmB treated hamsters. This improved efficacy was comparable to that observed with f-CNT conjugated and liposomal formulations of AmB, which suggests a general correlation between enhanced intracellular delivery and effectiveness of AmB.

A serious limitation of $\mathrm{AmB}$ in the treatment of VL has been its toxicity. In recent years various safer, lipid formulations of AmB (AmBisome, Abelcet, and Amphotec) have been commercialized, albeit at considerably increased expense. Carbon-based nanomaterials as scaffolds for AmB delivery are much more cost effective. ${ }^{20}$ However, cytotoxicity may set a substantial limitation when harnessing nanomaterials for biological settings. Both CNTs as well as some derivatives of graphene, particularly graphene oxide, have raised concerns of cytotoxicity and lack of hemocompatibility. ${ }^{26}$ Amine functionalization of graphene has been shown to be a safe alternative, and was thus employed in this study. Hence, quite predictably, the $\mathrm{CC}_{50}$ of $\mathrm{f}-\mathrm{Gr}-\mathrm{AmB}$ against J774A.1 cells was significantly more compared to that of AmB, with no evidence of cytotoxicity at clinically relevant concentrations. This was further affirmed by the minimal or no hepatic and renal toxicity in vivo.

In conclusion, treatment with $\mathrm{f}-\mathrm{Gr}-\mathrm{AmB}$ resulted in an improved therapeutic index between $\mathrm{IC}_{50}$ $(0.003 \pm 0.0004 \mu \mathrm{g} / \mathrm{mL})$ and $\mathrm{CC}_{50}(0.52 \pm 0.02 \mu \mathrm{g} / \mathrm{mL})$, hence increasing its safety profile and antileishmanial activity over conventional AmB. To the best of our knowledge, this is the first report demonstrating the potential use of $\mathrm{f}-\mathrm{Gr}$ as a vector for delivery of AmB. Our data evince the ascendancy of $\mathrm{f}-\mathrm{Gr}-\mathrm{AmB}$ over AmB, suggesting the potential of $\mathrm{f}-\mathrm{Gr}$ as a novel nanovector for AmB in the treatment of VL. Further studies are ongoing to compare f-Gr-AmB efficacy with that of liposomal AmB and to scrutinize its potential for oral administration. Despite these facts, better understanding of quantitative pharmacokinetics, biodistribution, in vivo behavior, as well as long term toxicology are acutely crucial and require further research.

\section{Acknowledgments}

We would like to thank Dr Vijay Kumar Prajapati, Dr Paresh Kulkarni, and all research scholars of the Infectious Disease
Research Laboratory, BHU for their kind help during the study, and Dr K Awsathi, Dr S Awasthi, and Dr H Raghuvanshi for the fruitful discussion on graphene synthesis. Shyam Lal Mudavath gives thanks to the University Grants Commission (UGC), New Delhi for providing a Junior Research Fellowship, and Mahe Talat is grateful to the Department of Science and Technology's (DST's) Women Scientists Scheme-A (WOS-A) for support.

This work was supported by DST (Unit on Nanoscience and Nanotechnology [UNANST], BHU) and the National Institute of Allergy and Infectious Diseases, National Institutes of Health (Tropical Medicine Research Center [TMRC] grant number P50AI074321). The funders had no role in study design, data collection and analysis, decision to publish, or preparation of the manuscript.

\section{Disclosure}

The authors report no conflicts of interest in this work.

\section{References}

1. Alvar J, Vélez ID, Bern C, et al. Leishmaniasis worldwide and global estimates of its incidence. PLoS One. 2012;7(5):e35671.

2. Thakur CP, Pandey AK, Sinha GP, Roy S, Behbehani K, Olliaro P. Comparison of three treatment regimens with liposomal amphotericin $\mathrm{B}$ (AmBisome) for visceral leishmaniasis in India: a randomized dosefinding study. Trans R Soc Trop Med Hyg. 1996;90(3):319-322.

3. Sundar S, Chatterjee M. Visceral leishmaniasis - current therapeutic modalities. Indian J Med Res. 2006;123(3):345-352.

4. Olliaro PL, Guerin PJ, Gerstl S, Haaskjold AA, Rottingen JA, Sundar S. Treatment options for visceral leishmaniasis: a systematic review of clinical studies done in India, 1980-2004. Lancet Infect Dis. 2005;5(12):763-774.

5. Sundar S, Singh A, Agarwal D, Rai M, Agrawal N, Chakravarty J. Safety and efficacy of high-dose infusions of a preformed amphotericin B fat emulsion for treatment of Indian visceral leishmaniasis. Am JTrop Med Hyg. 2009;80(5):700-703.

6. Egger SS, Meier S, Leu C, et al. Drug interactions and adverse events associated with antimycotic drugs used for invasive aspergillosis in hematopoietic SCT. Bone Marrow Transplant. 2010;45(7): 1197-1203.

7. Singh RK, Pandey HP, Sundar S. Visceral leishmaniasis (kala-azar): challenges ahead. Indian J Med Res. 2006;123(3):331-344.

8. Jain K, Jain NK. Novel therapeutic strategies for treatment of visceral leishmaniasis. Drug Discov Today. 2013;18(23-24):1272-1281.

9. Chellat F, Merhi Y, Moreau A, Yahia L. Therapeutic potential of nanoparticulate systems for macrophage targeting. Biomaterials. 2005;26(35):7260-7275.

10. Kansal S, Tandon R, Dwivedi P, et al. Development of nanocapsules bearing doxorubicin for macrophage targeting through the phosphatidylserine ligand: a system for intervention in visceral leishmaniasis. J Antimicrob Chemother. 2012;67(11):2650-2660.

11. Hillaireau H, Couvreur P. Nanocarriers' entry into the cell: relevance to drug delivery. Cell Mol Life Sci. 2009;66(17):2873-2896.

12. Manandhar KD, Yadav TP, Prajapati VK, et al. Antileishmanial activity of nano-amphotericin B deoxycholate. J Antimicrob Chemother. 2008;62(2):376-380.

13. Prajapati VK, Awasthi K, Gautam S, et al. Targeted killing of Leishmania donovani in vivo and in vitro with amphotericin $B$ attached to functionalized carbon nanotubes. J Antimicrob Chemother. 2011;66(4):874-879. 
14. Prajapati VK, Awasthi K, Yadav TP, Rai M, Srivastava ON, Sundar S. An oral formulation of amphotericin B attached to functionalized carbon nanotubes is an effective treatment for experimental visceral leishmaniasis. J Infect Dis. 2012;205(2):333-336.

15. Yang M, Yao J, Duan Y. Graphene and its derivatives for cell biotechnology. Analyst. 2013;138(1):72-86.

16. $\mathrm{Hu} \mathrm{W}$, Peng $\mathrm{C}$, Luo W, et al. Graphene-based antibacterial paper. ACS Nano. 2010;4(7):4317-4323.

17. Akhavan O, Ghaderi E, Rahighi R. Toward single-DNA electrochemical biosensing by graphene nanowalls. ACS Nano. 2012;6(4): 2904-2916.

18. Robinson JT, Tabakman SM, Liang Y, et al. Ultrasmall reduced graphene oxide with high near-infrared absorbance for photothermal therapy. J Am Chem Soc. 2011;133(17):6825-6831.

19. Kuila T, Bose S, Khanra P, Mishra AK, Kim NH, Lee JH. Recent advances in graphene-based biosensors. Biosens Bioelectron. 2011;26(12):4637-4648.

20. Sanchez VC, Jachak A, Hurt RH, Kane AB. Biological interactions of graphene-family nanomaterials: an interdisciplinary review. Chem Res Toxicol. 2012;25(1):15-34.

21. Novoselov KS, Fal'ko VI, Colombo L, Gellert PR, Schwab MG, Kim K. A roadmap for graphene. Nature. 2012;490(7419):192-200.

22. Rodriguez-Pérez L, Herranz MA, Martin N. The chemistry of pristine graphene. Chem Commun. 2013;49(36):3721-3735.

23. Zhu Y, Murali S, Cai W, et al. Graphene and graphene oxide: synthesis, properties, and applications. Adv Mater. 2010;22(35):3906-3924.

24. Shen H, Liu M, He H, et al. PEGylated graphene oxide-mediated protein delivery for cell function regulation. ACS Appl Mater Interfaces. 2012;4(11):6317-6323.

25. Sun X, Liu Z, Welsher K, et al. Nano-graphene oxide for cellular imaging and drug delivery. Nano Res. 2008;1(3):203-212.

26. Singh SK, Singh MK, Nayak MK, et al. Thrombus inducing property of atomically thin graphene oxide sheets. ACS Nano. 2011;5(6): 4987-4996.

27. Singh SK, Singh MK, Kulkarni PP, Sonkar VK, Grácio JJ, Dash D. Amine-modified graphene: thrombo-protective safer alternative to graphene oxide for biomedical applications. ACS Nano. 2012;6(3): 2731-2740
28. Maurya R, Mehrotra S, Prajapati VK, Nylén S, Sacks D, Sundar S. Evaluation of blood agar microtiter plates for culturing leishmania parasites to titrate parasite burden in spleen and peripheral blood of patients with visceral leishmaniasis. J Clin Microbiol. 2010;48(5): 1932-1934.

29. Staudenmaier L. Verfahren zur darstellung der graphitsäure. Berichte der Deutschen Chemischen Gesellschaft [A method for representation of graphite acid. Reports of the German Chemical Society]. 1898;31(2):1481-1487. German.

30. Besteman K, Lee JO, Wiertz FG, Heering HA, Dekker C. Enzymecoated carbon nanotubes as single-molecule biosensors. NanoLett. 2003;3(6):727-730.

31. Rana VK, Choi MC, Kong JY, et al. Synthesis and drug-delivery behavior of chitosan-functionalized graphene oxide hybrid nanosheets. Macromol Mater Eng. 2011;296(2):131-140.

32. Shan C, Yang H, Han D, Zhang Q, Ivaska A, Niu L. Water-soluble graphene covalently functionalized by biocompatible poly-L-lysine. Langmuir. 2009;25(20):12030-12033.

33. Schniepp HC, Li JL, McAllister MJ, et al. Functionalized single graphene sheets derived from splitting graphite oxide. J Phys Chem B. 2006;110(17):8535-8539.

34. Kaushik A, Khan R, Solanki PR, et al. Iron oxide nanoparticles-chitosan composite based glucose biosensor. Biosens Bioelectron. 2008;24(4): 676-683.

35. Dresselhaus MS, Jorio A, Hofmann M, Dresselhaus G, Saito R. Perspectives on carbon nanotubes and graphene Raman spectroscopy. Nano Lett. 2010;10(3):751-758.

36. Sundar S, More DK, Singh MK, et al. Failure of pentavalent antimony in visceral leishmaniasis in India: report from the center of the Indian epidemic. Clin Infect Dis. 2000;31:1104-1107.

37. De Jong WH, Borm PJ. Drug delivery and nanoparticles: Applications and hazards. Int J Nanomedicine. 2008;3(2):133-149. 


\section{Supplementary materials}

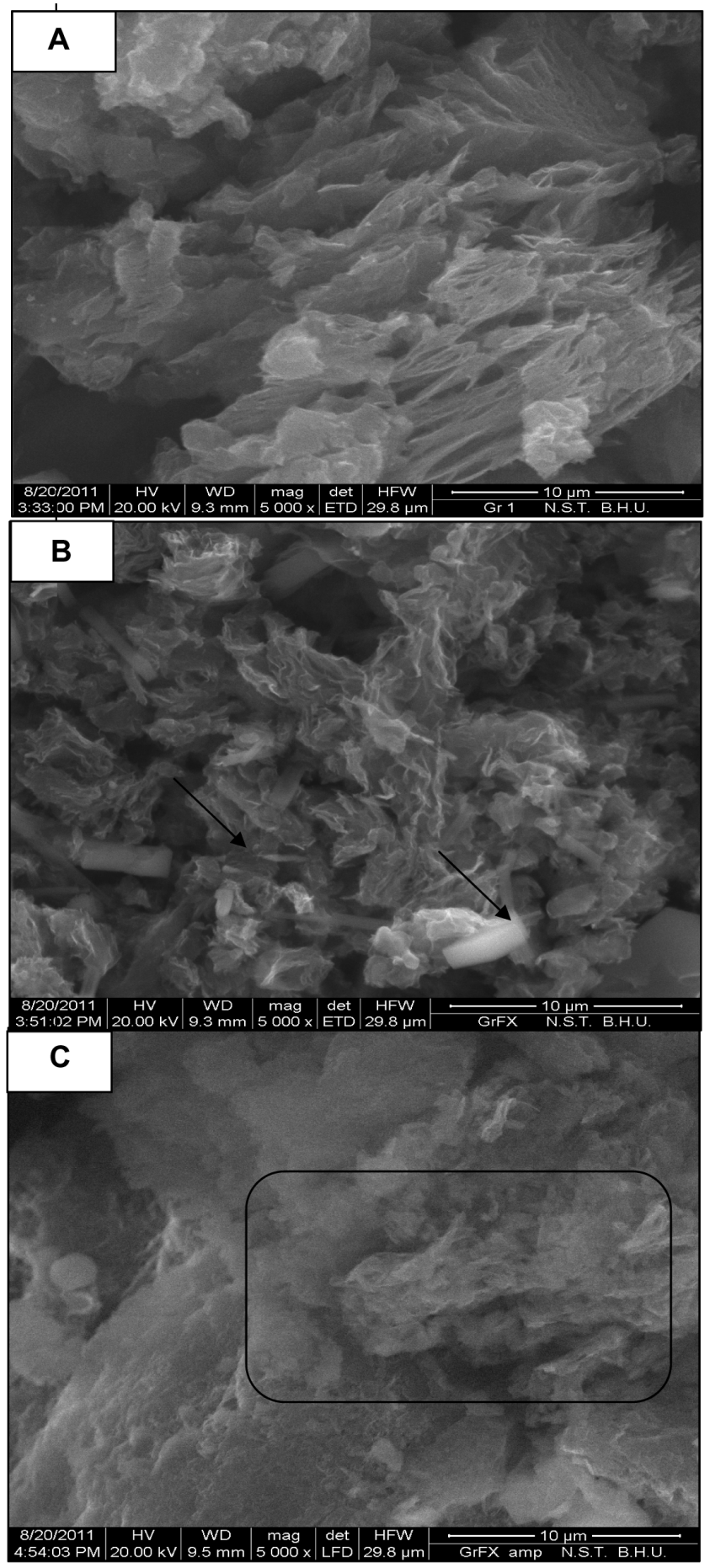

Figure SI SEM images of GS (A), f-Gr (B), and f-Gr-AmB (C).

Notes: (A) This figure shows clearly the typically flake like, slightly scrolled edged shapes of graphene sheet. (B) It is clearly visible from this figure that the small L-cysteine molecules are closely anchored on the surface of graphene sheets and held noncovalently (marked with arrows). (C) This figure illustrates the SEM image of f-Gr-AmB showing that a layer of drug was formed homogeneously (box area).

Abbreviations: SEM, scanning electron micrograph; GS, graphene sheets; $f-G r$, amine-modified graphene; f-Gr-AmB, a novel amphotericin B formulation as a conjugate with f-Gr. 
A
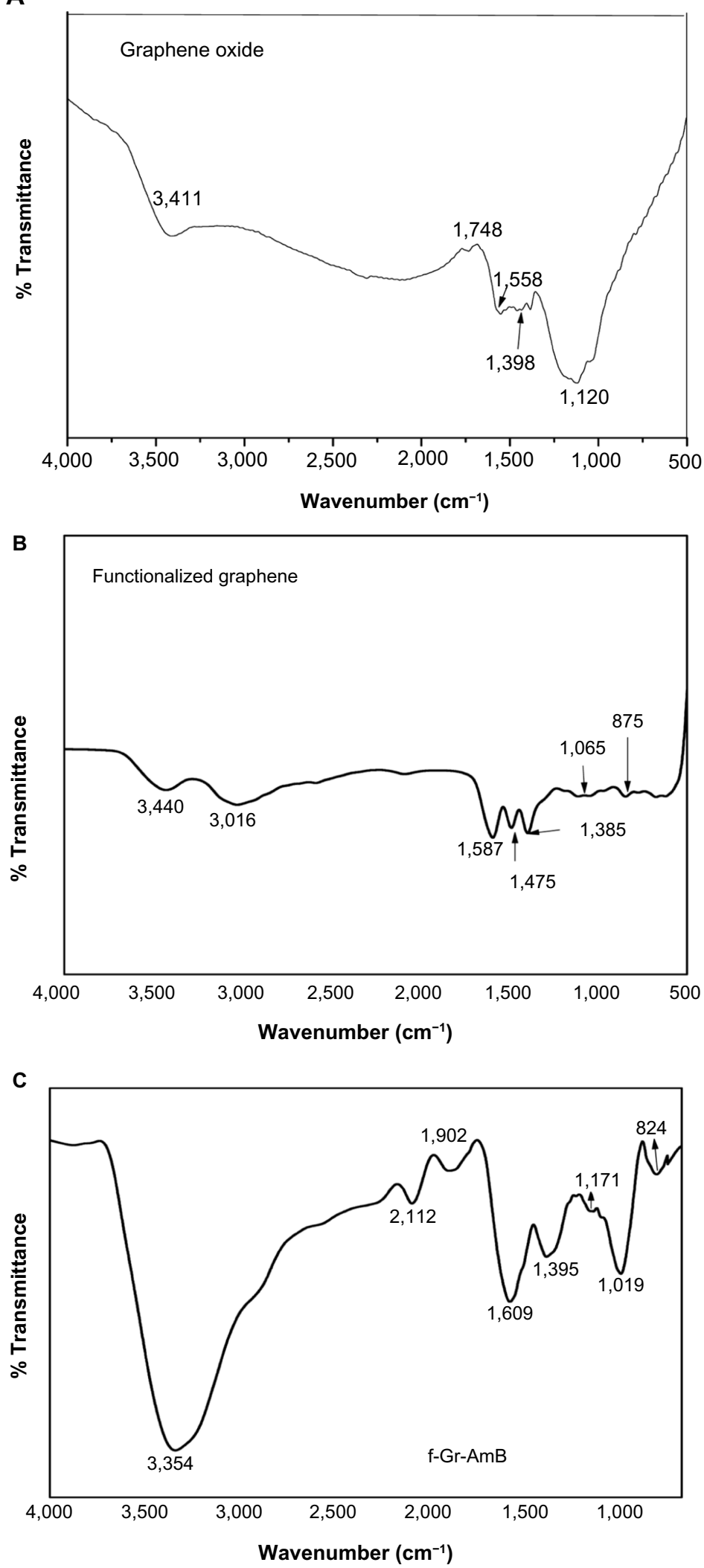

Figure S2 FTIR spectra of GS (A), f-Gr (B), and f-Gr-AmB (C).

Notes: Black arrows indicate the position of the peaks. (A) The absorption peak appearing at $1,585 \mathrm{~cm}^{-1}$ in terms of the known results is attributed to the vibration of carbon skeleton of graphene sheet. (B) An additional peak is observed at $3,016 \mathrm{~cm}^{-1}$ which is the stretching vibrations of the alkane group, while the presence of the peak at $1,475 \mathrm{~cm}^{-1}$ can be assigned to $\mathrm{N}-\mathrm{H}$ stretching vibrations of the amine group. (C) The peak at $1,019 \mathrm{~cm}^{-1}$ is associated with the stretching vibration mode of the hydroxyl group of the carboxylic group present in AmB.

Abbreviations: AmB, amphotericin B; FTIR, Fourier transform infrared; GS, graphene sheets, f-Gr, amine-modified graphene; f-Gr-AmB, a novel amphotericin B formulation as a conjugate with $\mathrm{f}-\mathrm{Gr}$. 
Drug Design, Development and Therapy

Dovepress

\section{Publish your work in this journal}

Drug Design, Development and Therapy is an international, peerreviewed open-access journal that spans the spectrum of drug design and development through to clinical applications. Clinical outcomes, patient safety, and programs for the development and effective, safe, and sustained use of medicines are a feature of the journal, which

has also been accepted for indexing on PubMed Central. The manuscript management system is completely online and includes a very quick and fair peer-review system, which is all easy to use. Visit $\mathrm{http}: / / \mathrm{www}$.dovepress.com/testimonials.php to read real quotes from published authors.

Submit your manuscript here: http://www.dovepress.com/drug-design-development-and-therapy-journal 